\title{
Über die Blutbewegung in den Kapillaren.
}

\author{
I. Mitteilung. \\ Registrierung der Strömungsgeschwindigkeit. \\ Von
}

Professor Dr. Adolf Basler, Tübingen.

(Mit 7 Textfiguren.)

Was für den ganzen Organismus die umgebende Luft und die Zufuhr der Nahrungsmittel, das bedeutet für die Zellen des Körpers das in den Haargefässen strömende Blut. Aus dem Blute reisst die Zelle den Sauerstoff an sich, aus dem Blute nimmt sie die zur Energieerzeugung notwendigen Brennstoffe auf. Von den Änderungen der Strömungsgeschwindigkeit in den verschiedenen Gefässen übt deshalb die in den Kapillaren vor sich gehende den unmittelbarsten Einfluss auf die Versorgung der Zellen aus. Zur Beurteilung der Ernährung eines bestimmten Gewebes ist es somit mindestens ebenso wichtig, die Strömungsverhältnisse in den zugehörigen Kapillaren zu kennen, als diejenigen in den grossen Arterien, zu deren Versorgungsgebiet ja durchaus heterogene Gewebe gehören.

Während zur Untersuchung der Strömungsgeschwindigkeit in den grösseren Gefässen naturgemäss verhältnismässig komplizierte Apparate notwendig sind, braucht man zur Beobachtung der Vorwärtsbewegung des Blutes in den Kapillaren nur ein Mikroskop.

Um mich bei dieser Art der Untersuchung nicht auf oberflächliche und besonders durchsichtige Organe beschränken zu müssen, bediene ich mich zur Beleuchtung eines entsprechend geformten Glasstäbchens ${ }^{1}$ ) (Lichtleiters), das unter das durchleuchtete Organ ge-

1) A. Basler, Über eine neue Methode zur mikroskopischen Untersuchung innerer Organe des lebenden Tieres im durchfallenden Licht nebst dem Versuch einer Theorie der das Licht leitenden Glasstäbe. Pflüger's Arch. Bd. 167 S. 228.1917. 
schoben wird, wodurch es gelingt, jedes Gewebe von unten nach oben mit hinreichend intensivem Licht $z u$ durchsetzen, so dass die Beobachtung im durchfallenden Licht ermöglicht wird.

Die Wirkung des Lichtleiters beruht darauf, dass die Lichtstrahlen an den Seiten des Glasstäbchens durch totale Reflexion zurückgeworfen werden, weshalb sie während ihrem ganzen Verlauf bis zu der Stelle, wo sie in den untersuchten Körperteil gelangen, in dem Glase bleiben müssen. Nun steckt aber der Lichtleiter mit seinem äussersten Ende im tierischen Gewebe. Bei der unvermeidlichen Berührung des Glases mit Gewebssaft muss ein Teil der Strahlen in diese Flüssigkeit, deren Brechungsexponent dem des Glases ziemlich ähnlich ist, gelangen und wird so dem durchleuchteten Organ entzogen. Trotzdem ist, wie meine Ergebnisse beweisen, die der Beobachtung zu gute kommende Lichtmenge auch so noch recht gross. Immerhin liesse sich eine Verbesserung der Methode dadurch erreichen, dass diejenigen im Tierkörper steckenden Teile des Lichtleiters, an welchen Reflexionen stattfinden, mit einem Spiegelbelag überzogen werden. Aber das gleichmässige Versilbern solch dünner Glasstäbchen ist sehr schwierig und demzufolge mit erheblichen Kosten verknüpft. $\mathrm{Da}$ ich gerade die Billigkeit des Lichtleiters bezw. die Möglichkeit, ihn jederzeit selbst herstellen zu können, als grossen Vorzug betrachte, so scbien es mir für die vorliegende Untersuchung nicht nötig, den Lichtverlust, der sich leicht durch Verstärkung der Lichtquelle ausgleichen lässt, durch Versilberung zu vermeiden. Für bestimmte Zwecke, die uns hier nicht interessieren, ist allerdings ein Spiegelbelag unerlässlich; ich werde darauf bei anderer Gelegenheit noch eingehend zu sprechen kommen.

Da die einfache Beobachtung nur eine mehr oder weniger zuverlässige Schätzung der Strömungsgeschwindigkeit gestattet, schien es mir wichtig, die Fortbewegung des Blutes auf photographischem Wege zu registrieren. Dabei beschränkte ich mich nicht auf die Haargefässe im engeren Sinn, sondern bezog auch kleinste Arterien und kleinste Venen mit in den Kreis der Untersuchung.

\section{Ausführung der Registrierung.}

Jede unmittelbare Registrierung von Bewegungen im lebenden Körper stösst begreiflicherweise auf gewisse Schwierigkeiten, die sich jedoch im vorliegenden Falle verhältnismässig leicht überwinden liessen. So ist schon die wichtigste Bedingung für eine graphische 
Registrierung, dass die Bewegung geradlinig verläuft, gewöhnlich in der Natur nicht erfüllt.

Bei meinen ersten Beobachtungen mit dem Lichtleiter war mir aufgefallen, dass die Blutgefässe im Sartorius des Frosches sich durch ihre regelmässige Anordnung auszeichnen ${ }^{1}$ ). Sie verlaufen in den Zwischenräumen zwischen den parallel angeordneten Muskelfasern und sind jedenfalls in einer Länge, wie sie für die mikroskopische Projektion in Betracht kommt, fast genau geradlinig. Diese Gefässe schienen mir für meine Versuche von vornherein wie geschaffen, und an ihnen wurden auch alle im folgenden beschriebenen Beobachtungen ausgeführt.

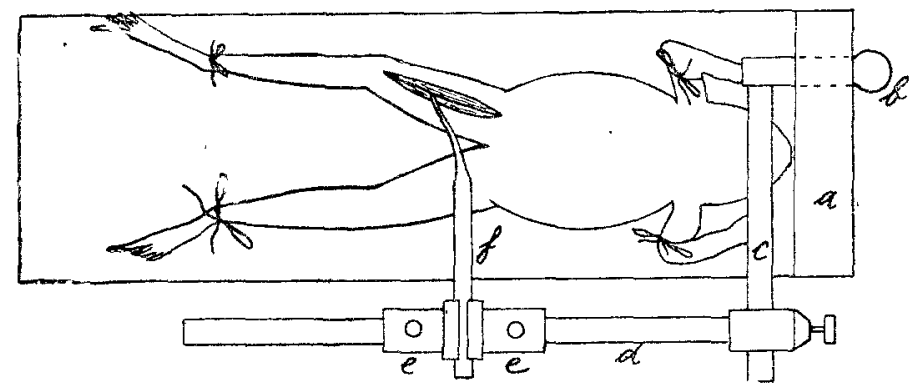

Fig. 1. Froschbrettchen mit Frosch von oben gesehen.

Zum Versuch wurde der kurarisierte Frosch auf einem Froschbrettchen mit dem Rücken nach unten aufgebunden, die Haut eines Schenkels wurde über dem M. sartorius gespalten und der Lichtleiter, wie bei meinen früheren Versuchen, unter den Muskel geschoben. Fig. 1 stellt von oben gesehen das Froschbrettchen mit dem darauf befestigten Frosch dar. Die Figur zeigt auch die Einrichtungen, mit deren Hilfe der Lichtleiter in der gewünschten Lage gehalten wird.

In dem Ansatz aus Holz a lässt sich ein Winkelstïck $b c$ um den Schenkel $b$ als Achse drehen. An dem Teile $c$ ist mit Hilfe

1) W. Spalteholz (Die Verteilung der Blutgefässe im Muskel. Abhandl. d. kgl, sächs. Gesellsch. d. Wissensch., mathem.-phys. Klasse Bd. 14 S. 507. 1888) reproduziert in Fig. 3,4 und 5 die Gefässverteilung im M. adductor magn. des Kaninchens. An diese Abbildungen erinnert, wenn man von den grösseren Gefässen absieht, der Froschsartorius; nur dass in ihm die Kapillaren noch regelmässiger verlaufen. 
einer kleinen Muffe das Stäbchen $d$ befestigt, das seinerseits die beiden durch Schrauben feststellbaren Schieber $e, e$ trägt. Zwischen die einander zugekehrten entsprechend geformten Endstücke dieser Schieber wird der Lichtleiter $f$ an der gewünschten Stelle eingeklemmt.

Das Froschbrettchen wird mit dem daraufliegenden Frosch genau in der gleichen Weise wie zur einfachen Beobachtung unter das Mikroskop (ich verwendete stets Leitz, Obj. 3, Okular 2) geschoben und der Muskelbezirk, welcher untersucht werden soll, eingestellt.

\section{Beleuchtung und Finstellung.}

Als Lichtquelle dient eine Bogenlampe $b$ Fig. 2, deren Lichtstrahlen durch eine grosse Kondensorlinse $e$ und ausserdem durch

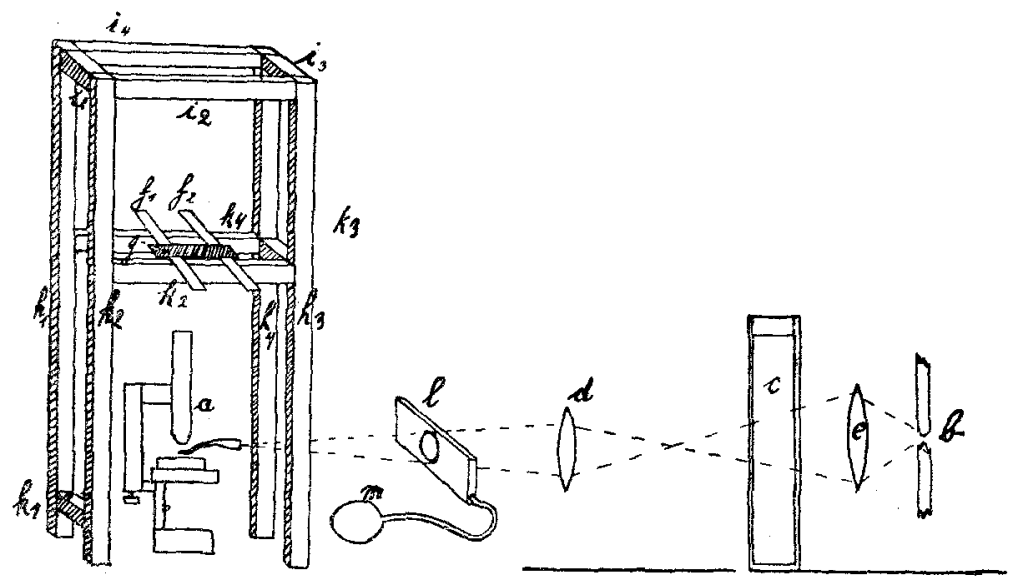

Fig. 2. Während das Holzgestell und der photographische Verschluss perspektivisch gezeichnet sind, sind die Kohlen der Bogenlampe, das Wassergefäss und die Linsen im Durchschnitt dargestellt.

eine zweite Konvexlinse $d$ hindurchgehen. Der mit Wasser gefüllte Glasbebälter $c$ hat den Zweck, einen Teil der Wärme zu absorbieren. Das Mikroskop ist mit a bezeichnet. Es wird so aufgestellt, dass der Konvergenzpunkt der Lichtstrahlen auf das freie Ende des Lichtleiters fällt. Durch entsprechende Einstellung des Mikroskops lässt sich in einer bestimmten Höhe über dem Ende des Tubus ein reelles Bild des Präparates erzeugen. Um dieses zu sehen, wird auf die beiden $12 \mathrm{~cm}$ über dem Okular liegenden Holzstäbchen $f_{1}$ und $f_{2}$ eine Mattglasscheibe $g$ gelegt. Wie die beiden Leisten $f_{1}$ und $f_{2}$ gelagert sind, soll später beschrieben werden. Auf dem so entstebenden 
Bilde heben sich besonders die verschiedenen Gefässe gut von der Umgebung ab. Bei heller Beleuchtung erkennt man sogar in ihnen die Blutzirkulation.

\section{Registriereinrichtung.}

Soll die Fortbewegung in einem bestimmten Gefäss registriert werden, dann darf natürlich nur dieses und nichts von der Umgebung sichtbar werden, was sich dadurch erreichen lässt, dass

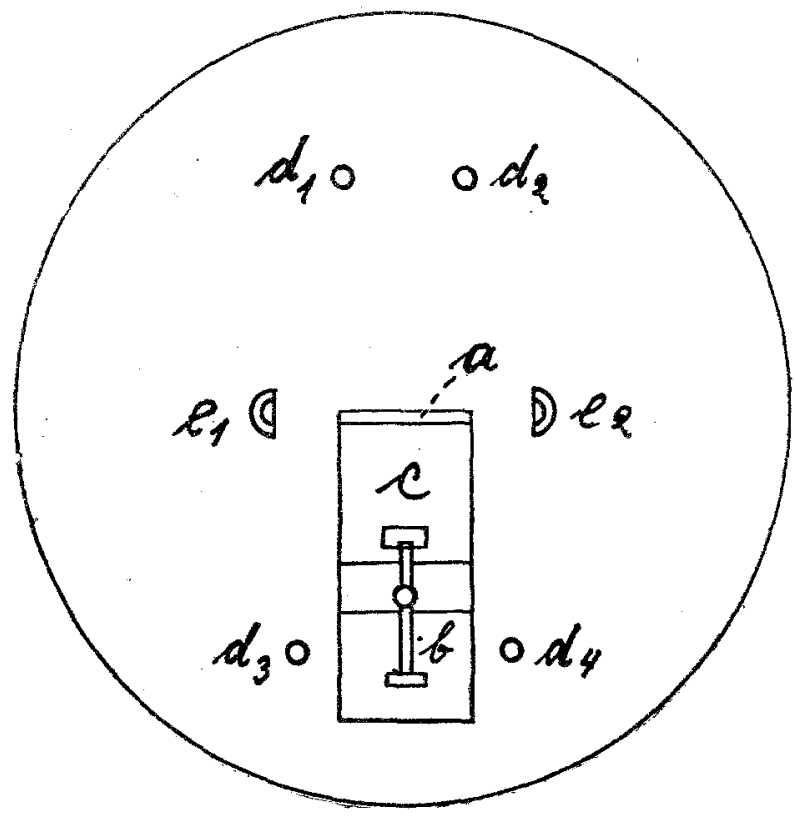

Fig. 3.

zwischen Mattscheibe und Mikroskop eine undurchsichtige Platte gebracht wird; in ihr befindet sich ein Spalt, dessen Ränder sich eng an das Gefäss anschliessen. Da aber die Abbildungen der Gefässe an im voraus unbekannten Stellen der Mattscheibe liegen und da sie in den versehiedensten Richtungen verlaufen, ist es notwendig, dass der Spalt je nach Bedarf verschoben und gedreht werden kann.

Den genannten Anforderungen wird in folgender Weise genügt. Über dem Mikroskop steht ein sehr stabil gebautes Gestell aus $3,5 \mathrm{~cm}$ dicken Holzstäbchen (vgl. Fig. 2 S. 137). Die vier senkrechten Stäbe $h_{1}$ bis $h_{4}$ sind $85 \mathrm{~cm}$ lang und werden durch die in zwei Etagen angeordneten $40 \mathrm{~cm}$ langen Querstäbe $i_{1}$ bis $i_{4}$ und $k_{2}$ bis 
$k_{4}$ zusammengehalten. Über die Stäbe $k_{2}$ und $k_{4}$ werden die schon oben erwähnten Stäbchen $f_{1}$ und $f_{2}$ gelegt, die, solange es sich nur um die Gewinnung eines Überblickes handelt, die Mattscheibe $g$ tragen. Zur Ausführung dèr Registrierung wird die Mattscheibe abgenommen und an ihre Stelle eine $7 \mathrm{~mm}$ dicke runde Metallplatte von $25 \mathrm{~cm}$ Durchmesser gelegt. Die Platte ist in Fig. 3 von oben gesehen dargestellt.

Das Wichtigste an ihr ist der $37 \mathrm{~mm}$ lange Spalt $a$, der mit Hilfe einer Schraube $b$, die an einem Schieber $c$ angreift, beliebig weit gestellt werden kann. Zur Einstellung wird der Spalt voll-

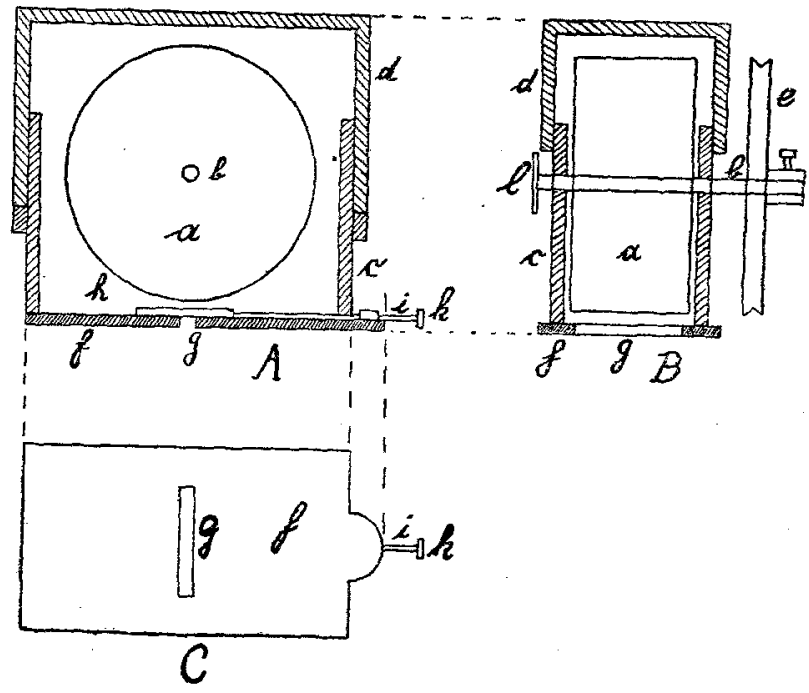

Fig. 4.

ständig geöffnet, so dass er etwa $1 \mathrm{~cm}$ weit ist. Kommt ausserdem über ihn die Mattscheibe $g$ Fig. 2 zu liegen, dann sieht man auf ihr wieder das Präparat abgebildet. Jetzt sucht man in dem Bild ein Gefäss, in welchem die Blutbewegung registriert werden soll, aus, dreht und schiebt die Scheibe so lange, bis die der Schraube $b$ gegenuberliegende Seite des Spaltes hart an dem einen Gefässrand anliegt. Mit Hilfe der Schraube $b$ wird hierauf der Spalt so weit verengert, dass die andere Seite des Gefässes von dem Schieher $c$ berührt wird.

Zur Abblendung von Seitenlicht wird zwischen Mikroskop und Platte eine $10 \mathrm{~cm}$ lange, vierkantige Hülse von $4 \mathrm{~cm}$ Durchmesser aus schwarzem Karton ( $b$ Fig. 5) gebracht, die unten durch ein $15 \mathrm{~cm}$ langes, schlauchähnliches Ansatzstück aus undurchsichtigem Stoff verlängert ist. Die Kartonhülse ist an einem Stativ ( $c$ Fig. 5) 
so hefestigt, dass sie an ihm gehoben und gesenkt werden kann. Am oberen Ende geht sie in einen kleinen Holzrahmen über, dessen obere Fläche mit schwarzem Samt überzogen ist, so dass sie sich dicht an die Metallplatte anschliesst. Das untere Ende des Stoffschlauches lässt sich mit Hilfe eines Bindfadens um den Tubus des Mikroskops zusammenziehen.

Einen besonderen Teil der Einrichtung bildet die $35 \mathrm{~mm}$ breite Trommel $a$ (Fig, 4) von $10 \mathrm{~cm}$ Durchmesser. Sie dreht sich in einem der Hauptsache nach aus Holz hergestellten Kasten $c$ mitsamt der durch sie gelegten Achse $b$. Der Kasten ist nach Art mancher photographischer Apparate mit schwarzer Leinwand überzogen und mit einem weit übergreifenden Deckel $d$ verschliessbar. Nach Abheben des Deckels ist die Trommel zugänglich und kann im Dunkelzimmer mit dem Film bespannt werden. Die Trommelachse $b$ ist nach der einen Seite hin verlängert und wird beim Versuch mit einem Rad $e$ versehen, durch welches von einem Uhrwerk aus mit Hilfe einer Transmission die Drehung der Trommel bewirkt wird.

Die untere Wand des Trommelgehäuses wird durch eine an den Rändern etwas vorstehende Metallplatte $f$ gebildet, welche in ihrer Mitte einen der Trommelachse parallelen $4 \mathrm{~cm}$ langen, $1 \mathrm{~cm}$ breiten Ausschnitt $g$ besitzt, der durch einen Schieber $h$ verschlossen werden kann. Der Schieber wird nit dem Stäbchen $i$, das am Ende einen Knopf $k$ trägt, bewegt. Bei der Aufuahme des Photogramms muss sich der Film selbstverständlich senkrecht zur Gefässachse, also auch senkrecht zu dem in der runden Platte (Fig. 3) befindlichen Spalt bewegen. Mit anderen Worten: Die Trommelachse muss parallel zum Spalt stehen. Damit nun der die Trommel enthaltende Kasten immer in die richtige Lage kommt, sind an der Metallplatte (Fig. 3) vier kurze nach oben stehende Metallstäbchen $d_{1}$ bis $d_{4}$ Fig. 3 angebracht, welche genau in Einschnitte an der Grundplatte $f$ des Trommelgehäuses (Fig. 4) passen. Ausserdem wird der vorstehende Teil der Platte $f$ beiderseits durch die zwei mit Kopf versehenen, drehbaren Haken $e_{1}$ und $e_{2}$ (Fig. 3) festgeklemmt. Da die Trommel bei jedem Versuch, entsprechend dem Verlauf des untersuchten Gefässes in einer anderen Richtung steht, so muss das sie treibende Uhrwerk ü ber der Trommel aufgestellt werden, damit die Uhrwerks- und Trommelachse ohne Schwierigkeit bei jeder Lage des untersuchten Gefässes parallel zueinander gerichtet werden 
können (vgl. Fig. 5). Zur Aufnahme des Uhrwerkes $n$ ist über den Stäbchen $i_{1}$ bis $i_{4}$ ein Brett $m$ befestigt. Dasselbe ist mit einem runden Aussehnitt versehen, durch den die Transmission $l l$ hindurchtreten und durch Vermittlung des Rades $g$ die in dem Kasten $e$ eingeschlossene Registriertrommel in Drehung versetzen kann. Die runde Metallplatte mit dem verstellbaren Spalt ist in Fig. 5 mit $d$ bezeichnet. $b$ stellt die auf $\mathbf{S}$. 139 beschriebene, an einem besonderen Stativ $c$ befestigte Hülse dar, die über den oberen Teil des Mikroskops gesteckt wird. In Fig. 5 sind ausserdem wie in Fig. 2 die senkrechten Stäbe des Holzgestelles mit $h$ und die Querstäbe mit $k$ und $i$ bezeichnet.

Natürlich darf bei jedem Versuch der Film nur während ein er Umdrehung der Trommel exponiert werden, sonst würden ja mehrere Photogramme übereinander gezeichnet. Um dies zu ermöglichen, wird in den Strahlengang des Lichtkegels ein photographischer Verschluss $l$ (Fig. 2) eingeschaltet, der durch Druck auf einen Gummiballon $m$ geöffnet bzw. geschlossen werden kann. So-

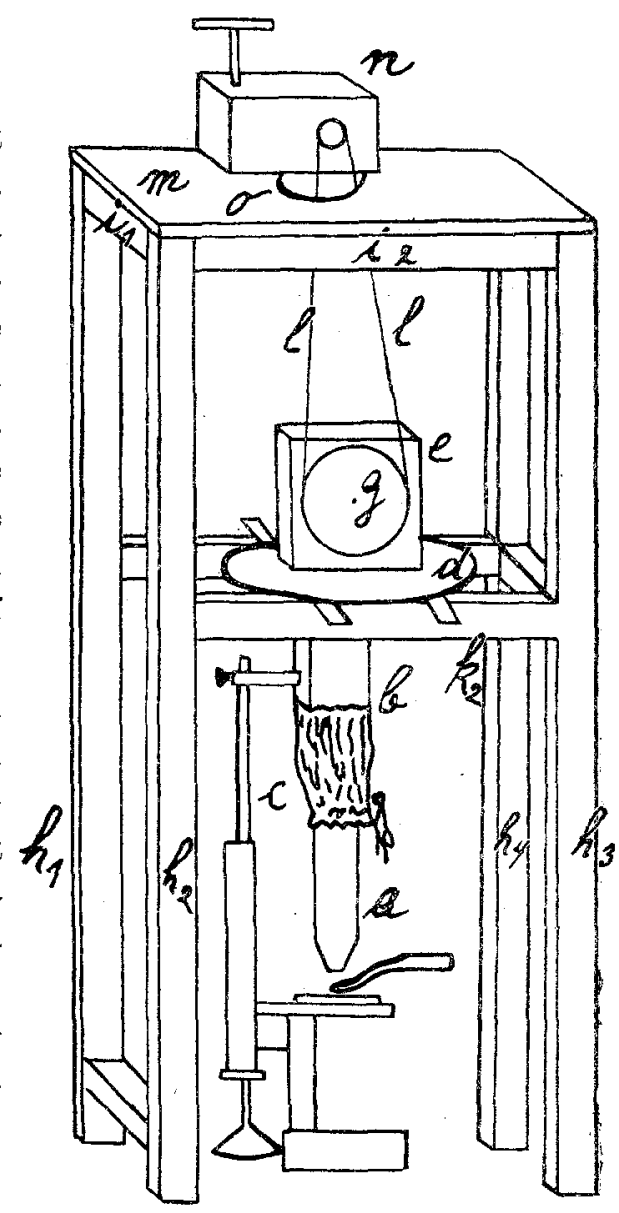

Fig. 5. Aufstellung zur Registrierung. Von dem Mikroskop ist zur Vereinfachung der Skizze nur der Tubus $a$ dargestellt.

lange die Trommel anläuft, bleibt der Verschluss abgesperrt; während dieser Zeit kann kein Licht auf das Ende des Lichtleiters fallen, und der über dem Spalt sich bewegende Filmstreifen wird nicht belichtet. Sobald die Trommel sich gleichmässig dreht, wird unmittelbar, ehe der Film den Spalt erreicht, geöffnet. Nach Ablaufen des Films wird das Licht durch Druck auf den Ballon sofort wieder abgeblendet. 
Um zur rechten Zeit öffnen und schliessen zu können, muss man über die Lage des. Filmstreifens in dem Kasten genau unterrichtet sein. Aus diesem Grunde ist an der dem Triebrade abgekehrten Seite der Trommelachse $b$, Fïg. $4 B$ ein kleines Rädchen $l$ angelötet, auf dem bestimmte Zeichen angebracht sind, an denen man die jeweilige Lage des Filmstreifens in dem Trommelgehäuse erkennen kann. Um die Verzeichnung von Zeitmarken auf dem Photogramm zu ermöglichen, ist an der S. 139 beschriebenen Hülse, nabe bei ihrem oberen Ende, eine kleine Öffnung angebracht, durch die der Zeiger eines Jaquet'schen Chronographen gesteckt werden kann, der seinen Schatten auf den Spalt wirft und so gleichzeitig mit dem Gefäss photographiert wird.

\section{Ergebnisse.}

Die mit der beschriebenen Versuchsanordnung erhaltenen Photogramme stellen Systeme von schrägen Linien dar. Dabei entspricht jeder helle Strich des Negativs einem Blutkörperchen (oder wenn es.

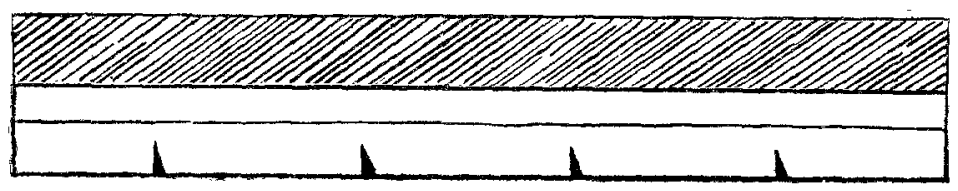

Fig. 6. Jeder schräge Strich entspricht einem Blutkörperchen. Zeitmarken $=$ Sekunden.

sich um grössere Gefässe handelt, einem Konglomerat von solchen). Man darf natürlich nicht erwarten, dass die Striche so kontrastreich werden wie in Kapillarelektrometerkurven. Aber immerhin sind sie deutlich genug für die Bestimmung ihrer Richtung und Länge. Namentlich im durchfallenden Licht heben sich die Linien gut von der Umgebung ab. Die Höhe der Kurve wird verhältnismässig kurz, weil das Gefässbild bald aus dem Spalt heraustritt. In Fig. 6 ist die Pause einer meiner Kurven wiedergegeben.

Da sich die Trommel senkrecht zur Fortbewegungsrichtung des Blutes dreht, so müssen die verzeichneten Linien um so steiler werden, je schneller sich die Blutkörperchen fortbewegen. Will man aus solchen Kurven die Geschwindigkeit der Blutkörperchen ermitteln, dann braucht man nur die Geschwindigkeit der Trommeldrehung und die Vergrösserung zu kennen. Die Trommelgéschwindig- 
keit ist aus den Zeitmarken unmittelbar zu ersehen, und die Vergrösserung betrug in allen meinen Versuchen 29,2:1. Durch Ausmessen der oben dargestellten Kurve lässt sich zum Beispiel ermitteln,

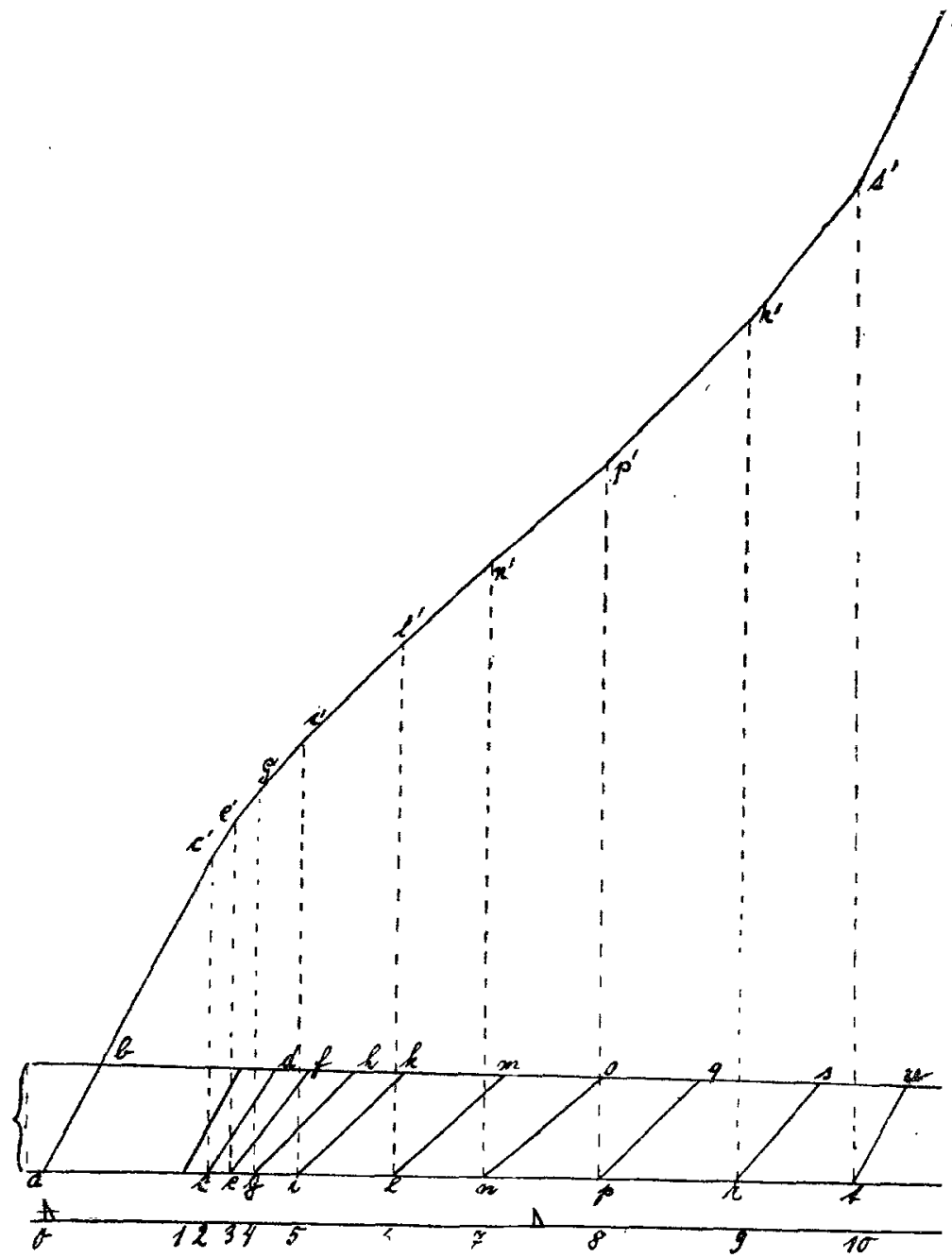

Fig. 7. Die schrägen Striche $a b, c d$, ef usw. stellen einige wenige der sämtlichen in dem Photogramm enthaltenen Striche dar. Auf der darunter liegenden Linie bedeuten die nach oben gerichteten Dreiecke Sekundenmarken. Die Zeiten, in denen jedesmal ein solcher Strich abgeht, sind mit $0,1,2$ usw. bezeichnet.

dass ein Strichpunkt in einer Sekunde um $14 \mathrm{~mm}$ gestiegen ist. Die wirkliche Bewegung der Blutkörperchen ist aber 29,2 mal kleiner; also bewegt sich ein Blutkörperchen in der Sekunde um $\frac{14}{29,2} \mathrm{~mm}$ $=0.5 \mathrm{~mm}$. 
Bei meinen Versuchen schwankten die Geschwindigkeiten je nach der Art des Gefässes zwischen 0,24 und 1,7 mm in der Sekunde. Die einzeluen Striche verlaufen geradlinig und parallel zueinander, wenn das Blut in dem untersuchten Gefäss sich gleichmässig fortbewegt. Ändert sich aber die Strömungsgeschwindigkeit in einem Gefäss pulsatorisch, wie dies bei kleinen Arterien der Fall ist, dann sind die Striche nicht mehr alle parallel, sondern es wechseln steilere Strichgruppen mit weniger steilen $a b$.

Ein solches Photogramm ist schematisch in Fig. 7 skizziert, wobei zunächst nur derjenige Teil der Zeichnung betrachtet werden wolle, der im Gebiete der Klammer \{ liegt.

Diese Kurve besagt, dass die Sekundengeschwindigkeit in der Zeit 1: $1,5 \mathrm{~mm}$ betrug, in der Zeit $7: 0,66 \mathrm{~mm}$. Die Geschwindigkeit zur Zeit 1 war die grösste (systolische), die zur Zeit 7 die kleinste (diastolische). Natürlich gibt jeder Strich die Geschwindigkeit eines anderen Blutkörperchens wieder. Will man sich ein Bild machen über die Fortbewegung eines einzelnen Blutkörperchens, dann muss man die verschiedenen Striche der. Kurve aneinanderreihen mit Hilfe einer Konstruktion, wie sie im oberen Teil der Fig. 7 durehgeführt ist.

Die Linie $a b c^{\prime} e^{\prime} l^{\prime}$ stellt dann die Fortbewegung eines Blutkörperehens dar. Sie entspricht der Kurve, welche durch Aufzeichnung der Blutströmung mittels der registrierenden Stromuhr von $\mathrm{H} u \mathrm{rthle}^{1}$ ) erbalten wird. Hier wie dort stellt die Ordinate eines bestimmten Punktes den zu dieser Zeit im ganzen zurückgelegten Weg dar. Soll aus dieser Kurve eine solche abgeleitet werden, bei der jede Ordinate der Geschwindigkeit, d. h. dem Weg in der Zeiteinheit proportional ist, etwa wie bei dem v. Kriesschen ${ }^{2}$ ) Flammentachogramm, dann müsste man die Linie $a b c^{\prime} e^{\prime} l^{\prime}$ umzeichnen, in der Art wie es $\mathrm{Fick}^{3}$ ) für das Plethysmogramm

1) K. Hürthle, Beschreibung einer registrierenden Stromuhr. Pflüger's Arch. Bd. 97 S. 193.1903.

2) J. v. Kries, Über ein nenes Verfahren zur Beobachtung der Wellenbewegung des Blutes. Arch. f. Anat. u. Physiol. 1887 S. 254, und Studien zur Pulslehre. J. Mo hr, Freiburg 1892.

3) A. Fick, Die Geschwindigkeitskurve in der Arterie des lebenden Menschen. (Braunmüller, Wien 1869.) Gesammelte Schriften Bd. 3 S. 550 (5\%4). Würzburg 1904. 
ausgeführt hat. Die Kurve Figur 7 stellt die Integralkurve des Tachogramms dar.

Die soeben kurz erwähnten Ergebnisse mögen den Gang und den Zweck der Untersuchungen etwas anschaulicher machen. Genauer soll auf sie erst in einer späteren Mitteilung eingegangen werden. Als die wesentliche Aufgabe der vorliegenden Publikation betrachte ich die Beschreibung der Methodik.

Was man bei meinen Untersuchungen sieht, bezw. aufschreibt, das ist die Bewegung der roten Blutkörperchen. Über die Strömung der Flüssigkeit können wir natürlich keinen Aufschluss erhalten. Mögen nun die Körperchen sich mit der Geschwindigkeit des axialen Blutstromes bewegen, wie es $\mathrm{Nicolai}^{-1}$ ) annimmt, oder nach der Frank'schen ${ }^{2}$ ) Auffassung mit der mittleren Geschwindigkeit der Strömung einer Kapillare; jedenfalls ist die Bewegung der.Blutkörperchen ein Ausdruck für die zurzeit in dem betreffenden Gefäss herrschende Strömungsgeschwindigkeit.

1) Fr. Nicolai, Die Mechanik des Kreislaufes. W. Nagel's Handbuch der Physiol. Bd. 1 S. 661 (765). 1909.

2) 0. Frank, Hämodynamik. R. Tigerstedt's Handbuch der physiol. Methodik Bd. 2 S. 1 (260). 1911. 